\title{
Generalized Split-Window Algorithm for Estimate of Land Surface Temperature from Chinese Geostationary FengYun Meteorological Satellite (FY-2C) Data
}

\author{
Bohui Tang ${ }^{1,2}$, Yuyun Bi ${ }^{3,4}$, Zhao-Liang $\mathrm{Li}^{1,3,{ }^{*}}$ and Jun Xia ${ }^{1}$
}

1 Key Laboratory of Water Cycle and Related Land Surface Processes, Institute of Geographic Sciences and Natural Resources Research, Chinese Academy of Sciences, Beijing, 100101, China E-mail: tangbh@igsnrr.ac.cn

2 Graduate University of Chinese Academy of Sciences

3 TRIO/LSIIT(UMR7005 CNRS)/ENSPS, Bld Sebastien Brant, BP10413, 67412 Illkirch, France E-mail: lizl@igsnrr.ac.cn

4 Institute of Agricultural Resources and Regional Planning, Chinese Academy of Agricultural Sciences, Beijing, 100086, China

\footnotetext{
* Author to whom correspondence should be addressed; E-mail: lizl@igsnrr.ac.cn
}

Received: 3 January 2008 / Accepted: 31 January 2008 / Published: 14 February 2008

\begin{abstract}
On the basis of the radiative transfer theory, this paper addressed the estimate of Land Surface Temperature (LST) from the Chinese first operational geostationary meteorological satellite-FengYun-2C (FY-2C) data in two thermal infrared channels (IR1, 10.3-11.3 $\mu \mathrm{m}$ and IR2, 11.5-12.5 $\mathrm{m}$ ), using the Generalized Split-Window (GSW) algorithm proposed by Wan and Dozier (1996). The coefficients in the GSW algorithm corresponding to a series of overlapping ranging of the mean emissivity, the atmospheric Water Vapor Content (WVC), and the LST were derived using a statistical regression method from the numerical values simulated with an accurate atmospheric radiative transfer model MODTRAN 4 over a wide range of atmospheric and surface conditions. The simulation analysis showed that the LST could be estimated by the GSW algorithm with the Root Mean Square Error (RMSE) less than $1 \mathrm{~K}$ for the sub-ranges with the Viewing Zenith Angle (VZA) less than $30^{\circ}$ or for the sub-rangs with VZA less than $60^{\circ}$ and the atmospheric WVC less than $3.5 \mathrm{~g} / \mathrm{cm}^{2}$ provided that the Land Surface Emissivities (LSEs) are known. In order to determine the range for the optimum coefficients of the GSW algorithm, the LSEs could be derived from the data in MODIS channels 31 and 32
\end{abstract}


provided by MODIS/Terra LST product MOD11B1, or be estimated either according to the land surface classification or using the method proposed by Jiang et al. (2006); and the WVC could be obtained from MODIS total precipitable water product MOD05, or be retrieved using Li et al.' method (2003). The sensitivity and error analyses in term of the uncertainty of the LSE and WVC as well as the instrumental noise were performed. In addition, in order to compare the different formulations of the split-window algorithms, several recently proposed split-window algorithms were used to estimate the LST with the same simulated FY-2C data. The result of the intercomparsion showed that most of the algorithms give comparable results.

Keywords: Land surface temperature, FY-2C data, Split-window algorithm.

\section{Introduction}

Land Surface Temperature (LST) is not only a good indicator of both the energy equilibrium of the Earth's surface and greenhouse effects, but also one of the key variables controlling fundamental biospheric and geospheric interactions between the Earth's surface and its atmosphere. It can play either a direct role such as when estimating longwave fluxes, or indirectly as when estimating latent and sensible heat fluxes [1,2]. Moreover, many other applications, such as evaportranspiration modeling [3, 4], estimating soil moisture [5], and climatic, hydrological, ecological and biogeochemical studying $[6,7]$ and so on, rely on the knowledge of LST. Consequently, it is crucial to have access to reliable estimates of surface temperature over large spatial and temporal scales. It is practically impossible to obtain such information from ground based measurements, whereas the satellite observations in the Thermal Infra-Red (TIR) appears to be very attractive since it can give access to global and temporal estimates of LST.

However, the retrieval of the LST from satellite data is a very difficult task because, besides the radiometric calibration and the cloud screening procedures, three types of corrections have to be made. They are emissivity corrections, atmospheric corrections and topography corrections [8]. Up to now, many algorithms for estimating the LST from satellite observations have been proposed. They may be roughly grouped into three categories: the single channel algorithm $[9,10]$, the split window algorithm $[11,12]$ and the triple window algorithm [13].

The single channel method is a simple inversion of the radiative transfer equation providing that the Land Surface Emissivities (LSEs) and the atmospheric profiles are known in advance. The triple window method combines two thermal window channels and one middle infrared channel to estimate the LST for nighttime satellite observations. The split window method is used to retrieve the LST based on the differential water vapor absorption in two adjacent infrared channels. This method was firstly proposed by McMillin (1975) [11] to estimate sea surface temperature from satellite measurements. Since then, a variety of split window algorithms have been developed and modified to retrieve LST, and, currently, most of them have been successfully applied to the LST retrieval from the 
data observed by the AVHRR, MODIS, and Spinning Enhanced Visible and Infrared Imager (SEVIRI) instruments [8, 14-22].

The FengYun-2C (FY-2C), a geostationary meteorological satellite developed by Shanghai Academy of Space Flight Technology (SAST, also known as $8^{\text {th }}$ Space Academy) and China Academy of Space Technology (CAST, also know as $5^{\text {th }}$ Space Academy) and operated by China Meteorological Administration (CMA), was launched on 19 October 2004 and is becoming fully operational in 2006. The FY-2C is the Chinese first operational meteorological satellite, which is also the fourth satellite of the FY series and is located above the Equator at longitude $105^{\circ} \mathrm{E}$, and some $35,800 \mathrm{~km}$ away. The objective of the mission is to monitor the temperature and the clouds above China and neighboring areas and also to provide meteorological information for the Asia-Pacific region. The upgraded Stretched-Visible and Infrared Spin-Scan Radiometer (S-VISSR) is one of the major payloads onboard the FY-2C. This optical imaging radiometer consists of one visible channel and four infrared channels. The characteristics of the instrument are shown in Table 1. It can acquire one full disc image covering the Earth surface from $60^{\circ} \mathrm{N}$ to $60^{\circ} \mathrm{S}$ in latitude and from $45^{\circ} \mathrm{E}$ to $165^{\circ} \mathrm{E}$ in longitude per hour and $30 \mathrm{~min}$ per acquisition for flood season.

The work presented in this paper aims to retrieve LST from the FY-2C satellite data in two thermal infrared channels (IR1, 10.3-11.3 $\mu \mathrm{m}$ and IR2, 11.5-12.5 $\mu \mathrm{m}$ ), using the Generalized Split-Window (GSW) algorithm proposed by Wan and Dozier (1996) [21]. Section 2 describes the theory associated with the LST retrieval using the GSW algorithm and presents the algorithm development for FY-2C data. Section 3 gives the results and the numerical values of the coefficients in the GSW algorithm. The sensitivity and error analyses in term of the uncertainty of the LSE and Water Vapor Content (WVC) in the atmosphere as well as the instrumental noise are also presented in this section. In addition, in order to compare the different formulations of the split-window algorithms, this section gives the intercomparsion of the LSTs estimated by several split-window algorithms. Section 4 gives an example of retrieving LST from FY-2C satellite data. The Conclusion is drawn in Section 5.

Table 1. Specifications of S-VISSR channels: spectral range and spatial resolutions.

\begin{tabular}{|l|l|l|l|}
\hline Channel no. & Channel name & Spectral range $(\boldsymbol{\mu m})$ & Spatial resolution $(\mathbf{k m})$ \\
\hline 1 & IR1 & $10.3-11.3$ & 5 \\
\hline 2 & IR2 & $11.5-12.5$ & 5 \\
\hline 3 & IR3 & $6.3-7.6$ & 5 \\
\hline 4 & IR4 & $3.5-4.0$ & 5 \\
\hline 5 & VIS & $0.55-0.90$ & 1.25 \\
\hline
\end{tabular}

\section{Theory}

\subsection{Radiative transfer for split-window algorithm}

On the basis of the radiative transfer theory, for a cloud-free atmosphere under thermodynamic equilibrium, the channel radiance $B_{i}\left(T_{i}\right)$ measured at the Top Of the Atmosphere (TOA) in a Thermal Infra-Red (TIR) channel of the sensor onboard the satellite, is given with a good approximation as [23] 


$$
B_{i}\left(T_{i}\right)=\varepsilon_{i} B_{i}\left(T_{s}\right) \tau_{i}+R_{a t m_{-} i}^{\uparrow}+\left(1-\varepsilon_{i}\right) R_{a t m_{-} i}^{\downarrow} \tau_{i}
$$

where $T_{i}$ is the channel brightness temperature observed in channel $i$ at the TOA, $B_{i}$ is the Planck function, $B_{i}\left(T_{s}\right)$ is the radiance measured if the surface is a black body with surface temperature $T_{s}$, $\varepsilon_{i}$ is the channel emissivity in channel $i, \tau_{i}$ is the total atmospheric transmittance along the target to sensor path in channel $i, R_{\text {atm } i}^{\uparrow}$ is the thermal path atmospheric upwelling radiance in channel $i$, and $R_{\text {atm_i }}^{\downarrow}$ is the channel downwelling atmospheric radiance from the whole hemisphere in channel $i$. The first term on the right hand side of Eq. (1) represents the surface emission that is attenuated by the atmosphere. The second term represents the upwelling atmosphere emission toward the sensor and the third term represents the downwelling atmosphere emission that is reflected by the surface and reaches the sensor.

Inverting Eq. (1), one can get

$$
T_{s}=B^{-1}\left[\frac{B_{i}\left(T_{i}\right)-R_{a t m_{-} i}^{\uparrow}-\left(1-\varepsilon_{i}\right) R_{a t m_{-} i}^{\downarrow} \tau_{i}}{\varepsilon_{i} \tau_{i}}\right]
$$

in which $B^{-1}$ is the inverse of the Planck function. Once the channel emissivity $\varepsilon_{i}$ is known, there are two ways to estimate the LST from satellite data. One is to use Eq. (2) with atmospheric radiative transfer model such as MODTRAN 4 [24] or 4A/OP [25], if the atmospheric profile is available from either conventional radiosoundings or satellite soundings. The other is to employ the split-window algorithm developed on the basis of the differential water vapor absorption in two adjacent infrared channels (McMilin, 1975) [11] if the atmospheric profile is not available.

As S-VISSR sensor onboard FY-2C has two adjacent thermal infrared channels (IR1 and IR2), the GSW algorithm proposed by Wan and Dozier [21] is adopted to estimate the LST from FY-2C satellite data. According to GSW algorithm, the LST can be expressed as

$$
T_{s}=a_{0}+\left(a_{1}+a_{2} \frac{1-\varepsilon}{\varepsilon}+a_{3} \frac{\Delta \varepsilon}{\varepsilon^{2}}\right) \frac{T_{i}+T_{j}}{2}+\left(a_{4}+a_{5} \frac{1-\varepsilon}{\varepsilon}+a_{6} \frac{\Delta \varepsilon}{\varepsilon^{2}}\right) \frac{T_{i}-T_{j}}{2}
$$

with $\varepsilon=\left(\varepsilon_{i}+\varepsilon_{j}\right) / 2$ and $\Delta \varepsilon=\varepsilon_{i}-\varepsilon_{j}$.

where $T_{i}$ and $T_{j}$ are the TOA brightness temperatures measured in channels $i(11.0 \mu \mathrm{m})$ and $j(12.0$ $\mu m$ ), respectively; $\varepsilon_{i}$ and $\varepsilon_{j}$ are, respectively, the land surface emissivities in channels $i$ and $j ; \varepsilon$ is the averaged emissivity; $\Delta \varepsilon$ is the emissivity difference between the two adjacent channels; and $a_{0}-a_{6}$ are unknown coefficients which will be derived in the following from simulated FY-2C data..

\subsection{Algorithm development for $F Y-2 C$}

So far, as there is no available database of in situ LST measurements in coincidence with the FY-2C overpasses, the only possible way to obtain the coefficients in Eq. (3) is to use numerical simulation for establishing the database used in the statistical regression. To this end, the atmospheric radiative 
transfer model MODTRAN 4 was used to simulate the TOA radiance with the appropriate thermal infrared channel response function of the S-VISSR onboard FY-2C.

Keeping in mind that a practical LST algorithm should accommodate atmospheric variations wide enough to cover all possible real situations, two radiosonde observation databases were taken into account in our simulation. One is the latest version of the Thermodynamic Initial Guess Retrieval (TIGR) database TIGR2002, which was constructed by the Laboratoire de Meteorologie Dynamique (LMD) and represents a worldwide set of atmospheric situations (2311 radisoundings) from polar to tropical atmosphere with varying water vapor amounts ranging from 0.1 to $8 \mathrm{~g} / \mathrm{cm}^{2}$ (http://ara.lmd.polytechnique.fr/htdocs-public/products/TIGR/TIGR.html). The other is the six standard atmospheric profiles (tropical, mid-latitude summer, mid-latitude winter, sub-arctic summer, sub-arctic winter, and US76) stored in the MODTRAN 4. For LST retrieval, we only consider atmospheric variation in clear-sky conditions. Consequently, the profiles with relative humidity at one of levels greater than $90 \%$ in TIGR2002 were discarded as this seldom happens under clear-sky conditions. Therefore, 1413 representative atmospheric situations were extracted from TIGR2002. Figure 1 shows a plot of the atmospheric Water Vapor Content (WVC) as function of the atmospheric temperature $T_{0}$ in the first boundary layer of these selected atmospheres. As shown in this figure, the $T_{0}$ varies from $231 \mathrm{~K}$ to $315 \mathrm{~K}$ and the atmospheric WVC changes from $0.06 \mathrm{~g} / \mathrm{cm}^{2}$ to $6.44 \mathrm{~g} / \mathrm{cm}^{2}$.

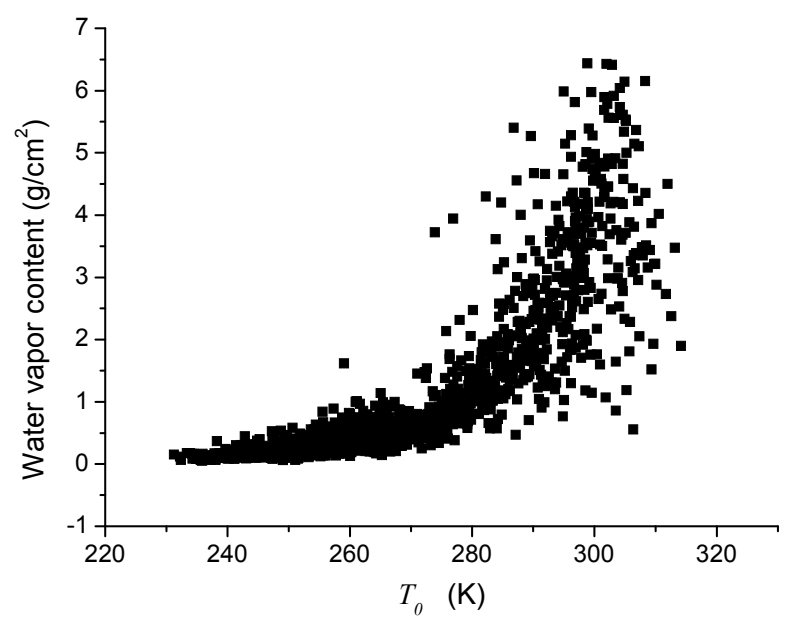

Figure 1. Plot of the atmospheric water vapor content as function of atmospheric temperature $T_{0}$ in the first boundary layer of the selected 1413 atmospheric profiles in TIGR2002.

Taking into account the angular dependence of the TOA radiance, six different Viewing Zenith Angles (VZAs) $\left(0^{\circ}, 33.56^{\circ}, 44.42^{\circ}, 51.32^{\circ}, 56.25^{\circ}, 60^{\circ}\right)$ varying from $0^{\circ}$ to $60^{\circ}$ were used in MODTRAN simulations. With the VZAs and the radiosoundings mentioned above as MODTRAN input, we can obtain the channel atmospheric parameters $\left(\tau_{i}, R_{\text {atm } i}^{\uparrow}, R_{\text {atm } i}^{\downarrow}\right)$ with spectral integration of the channel response function for each VZA and each atmospheric profile.

In addition, in order to make the simulation more representatives, the reasonable variations of LST are varied in a wide range according to the atmospheric temperature $T_{0}$ in the first boundary layer of 
the atmospheric profiles used. That is, LST varies from $T_{0}-5 \mathrm{~K}$ to $T_{0}+15 \mathrm{~K}$ in steps of $5 \mathrm{~K}$ for $T_{0} \geq 290 \mathrm{~K}$, and from $T_{0}-5 \mathrm{~K}$ to $T_{0}+5 \mathrm{~K}$ in steps of $5 \mathrm{~K}$ for $T_{0}<290 \mathrm{~K}$. Moreover, considering the most land covers, the averaged emissivity $\varepsilon$ varying from 0.90 to 1.0 with a step of 0.02 and the emissivity difference $\Delta \varepsilon$ from -0.025 to 0.015 with a step of 0.005 , were used in our simulation [21].

Then for a given LST, in combination with the atmospheric parameters $\left(\tau_{i}, R_{a t m_{-} i}^{\uparrow}, R_{a t m_{-} i}^{\downarrow}\right)$, LST $\left(T_{s}\right)$ and $\operatorname{LSE}\left(\varepsilon_{i}\right)$, the channel brightness temperature $T_{i}$ at the TOA can be determined according to Eq. (1) with the inverse of Planck's law. At this stage, the $T_{s}$ is directly related to the TOA measured brightness temperatures $T_{i}$ and $T_{j}$. The coefficients $a_{0}-a_{6}$ in Eq. (3) can be obtained through statistical regression method. In total, for the TIGR2002 database and the six standard MODTRAN 4 atmospheres, 261738 different situations were obtained for each VZA.

\section{Results and Analysis}

\subsection{GSW algorithm coefficients}

In order to determine the coefficients $a_{0}-a_{6}$ in Eq. (3), Wan and Dozier (1996) [21] divided the averaged emissivity, atmospheric WVC and atmospheric surface temperature $\left(T_{0}\right)$ into several tractable sub-ranges for improving the fitting accuracy. Taking into account the fact that the S-VISSR sensor onboard FY-2C has no atmospheric sounding channels, the atmospheric surface temperature is not simultaneously available, and thus it will be substituted in this work for the determination of the coefficients in Eq. (3) by the approximate Land Surface Temperature (LST).

For different values of the numerical experiments, in order to improve the accuracy of the retrieval LST, for each VZA as done in [21], the averaged emissivity was divided into two groups: one varies from 0.90 to 0.96 and the other ranges from 0.94 to 1.0. The WVC was divided into six sub-ranges with an overlap of $0.5 \mathrm{~g} / \mathrm{cm}^{2}$ : [0, 1.5], [1.0, 2.5], [2.0, 3.5], [3.0, 4.5], [4.0, 5.5], and [5.0, 6.5] g/cm ${ }^{2}$. The LST, $T_{s}$, was divided into five sub-ranges with an overlap of $5 \mathrm{~K}: T_{s} \leq 280 \mathrm{~K}, 275 \leq T_{s} \leq 295 \mathrm{~K}$, $290 \leq T_{s} \leq 310 \mathrm{~K}, 305 \leq T_{s} \leq 325 \mathrm{~K}, T_{s} \geq 320 \mathrm{~K}$. Then, the coefficients $a_{0}-a_{6}$ in Eq. (3) can be obtained through statistical regressions method for each VZA and each sub-range.

As an example, Figure 2 displays the coefficients of the GSW algorithm as functions of the secant VZA for the sub-range with WVC from $1.0 \mathrm{~g} / \mathrm{cm}^{2}$ to $2.5 \mathrm{~g} / \mathrm{cm}^{2}$, and LST varying from $290 \mathrm{~K}$ to $310 \mathrm{~K}$ for the two emissivity groups. As shown in this figure, the coefficients $a_{0}-a_{6}$ for other VZAs can be linearly interpolated in function of the secant VZA. Similar results are obtained for the other subranges. 


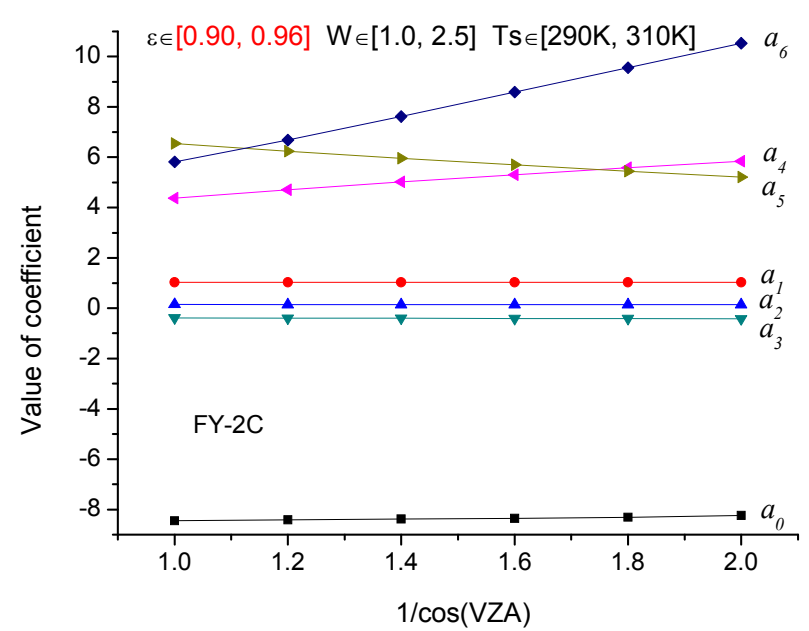

(a)

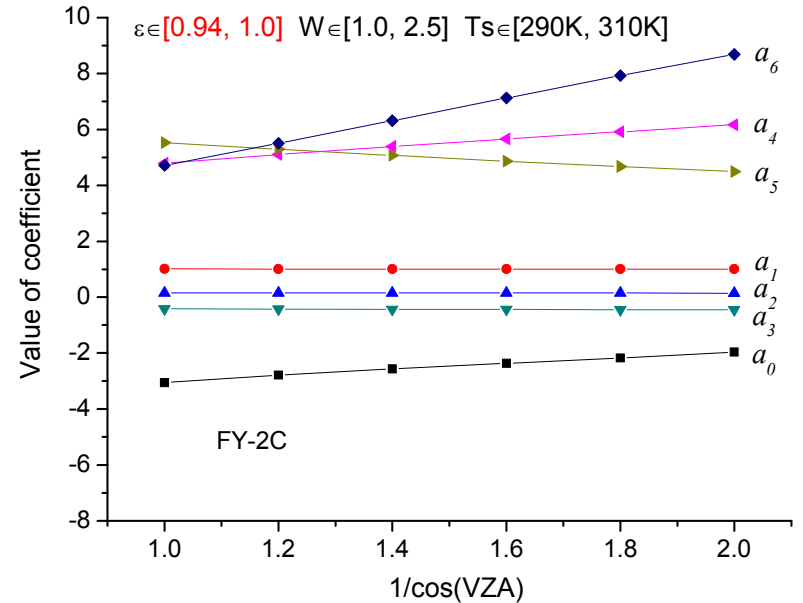

(b)

Figure 2. Coefficients of the generalized split-window algorithm for the sub-range with LST varying from $290 \mathrm{~K}$ to $310 \mathrm{~K}$, and WVC from $1.0 \mathrm{~g} / \mathrm{cm}^{2}$ to $2.5 \mathrm{~g} / \mathrm{cm}^{2}$. (a) for $\varepsilon \in[0.90,0.96]$ and (b) for $\varepsilon \in[0.94,1.0]$

\subsection{Estimation of LST}

Figure 3 shows, respectively, the histogram of the difference between the actual $T_{s}$ and the $T_{s}$ estimated using GSW algorithm with the coefficients corresponding to the sub-range $W V C \in[1.0,2.5]$, and $T_{s} \in[290 K, 310 K]$ for two different emissivity groups and $\mathrm{VZA}=0^{\circ}$. The Root Mean Square Errors (RMSEs) between the actual and estimated $T_{s}$ is $0.37 \mathrm{~K}$ for the emissivity group $\varepsilon \in[0.94,1.0]$, and $0.48 \mathrm{~K}$ for the other emissivity group $\varepsilon \in[0.90,0.96]$. Similar results were obtained for the other VZAs.

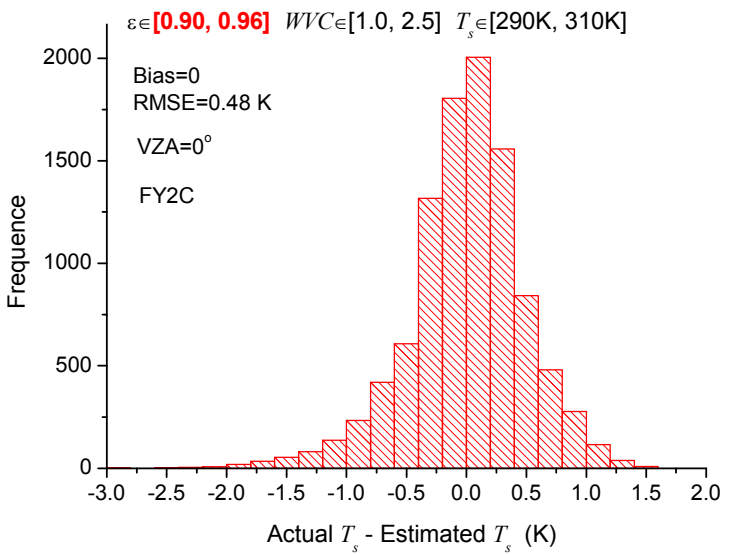

(a)

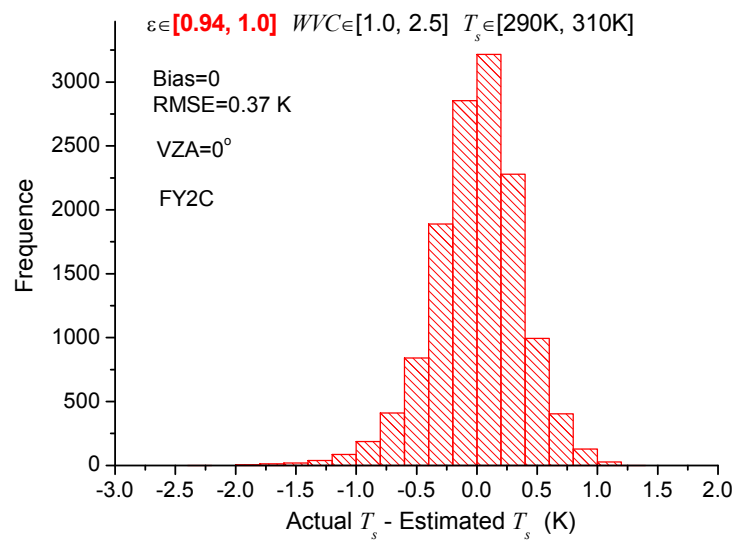

(b)

Figure 3. Histogram of the difference between the actual and estimated $T_{s}$ for the subrange with LST varying from $290 \mathrm{~K}$ to $310 \mathrm{~K}$, and WVC from $1.0 \mathrm{~g} / \mathrm{cm}^{2}$ to $2.5 \mathrm{~g} / \mathrm{cm}^{2}$. (a) for $\varepsilon \in[0.90,0.96]$ and (b) for $\varepsilon \in[0.94,1.0]$. 

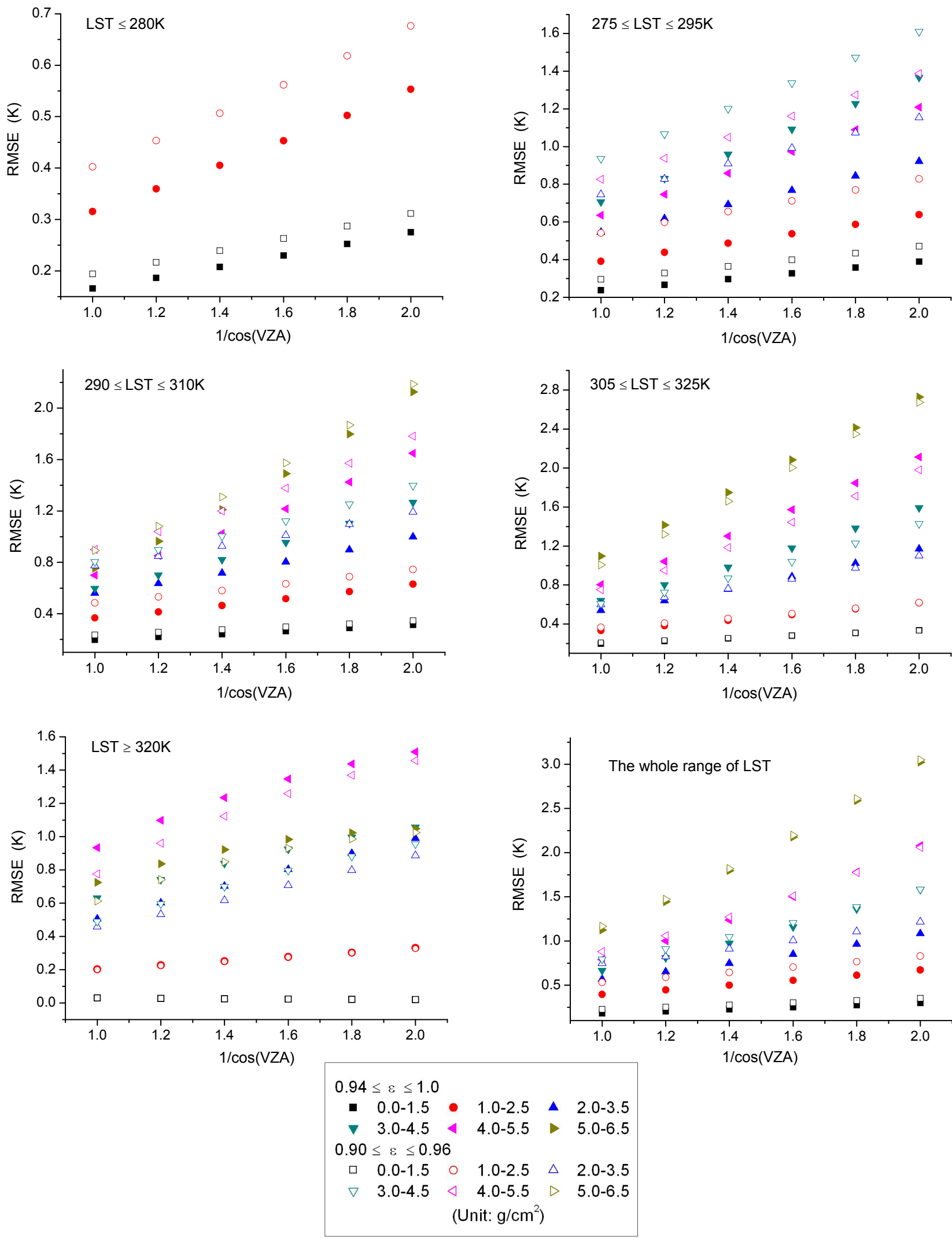

Figure 4. RMSEs between the actual and estimated $T_{s}$ as functions of the secant VZA for different sub-ranges in two different emissivity groups. 
In addition, Figure 4 gives the RMSEs between the actual and estimated $T_{s}$ as functions of the secant VZA for the two emissivity groups with different sub-ranges. Taking into account that, in reality, the lower LST is usually accompanied with much less WVC, as shown in figure 1, Therefore, for the LST less than $280 \mathrm{~K}$, the maximum WVC is $2.5 \mathrm{~g} / \mathrm{cm}^{2}$, while for the LST between $275 \mathrm{~K}$ and $295 \mathrm{~K}$, the maximum WVC is $5.5 \mathrm{~g} / \mathrm{cm}^{2}$.

From figure 4, one can see that the RMSEs increase with the increase of the VZA. The RMSEs are less than $1 \mathrm{~K}$ for all sub-ranges with the VZA less than $30^{\circ}$, or for all sub-ranges with the VZA less than $60^{\circ}$ and the WVC less than $3.5 \mathrm{~g} / \mathrm{cm}^{2}$. The RMSEs increase dramatically with the increase of the VZA when the WVC larger than $3.0 \mathrm{~g} / \mathrm{cm}^{2}$, with the maximum RMSE of $2.7 \mathrm{~K}$ for the sub-range $\varepsilon \in[0.94,1.0], W V C \in[5.0,6.5]$, and $T_{s} \in[305 K, 325 K]$, for $\mathrm{VZA}=60^{\circ}$.

It should be pointed out here that, in practice, the LST is estimated in two steps for actual satellite data. Firstly, approximate LSTs are estimated using Eq. (3) with the coefficients derived for the whole range of LST providing that the sub-ranges of emissivity and WVC are known, and then more accurate LSTs are estimated once again using Eq. (3), but with the coefficients $a_{0}-a_{6}$ corresponding to the sub-range of LST which is determined according to the approximate LST obtained in the first step. Figure 4 also shows the RMSEs between the actual $T_{s}$ and the $T_{s}$ estimated with the coefficients obtained for the whole range of LST.

Keeping in mind that the GSW algorithm also requires LSE and WVC as model input, the following section will present the determination of these two parameters.

\subsection{Determination of the LSES}

The LSEs in channels IR1 and IR2 of S-VISSR can be estimated from the LSEs in channels 31 $(11 \mu \mathrm{m})$ and $32(12 \mu \mathrm{m})$ of MODIS provided by the MODIS LST product MOD11B1 at $5 \mathrm{~km}$ resolution. To determine the emissivity relationship between S-VISSR channels and MODIS 31 and 32 channels, two spectral databases, one from the University of California Santa Barbara (UCSB) (http://www.icess.ucsb.edu/modis/EMIS/html/em.html) and the other from the Johns Hopkins University (JHU) (http://speclib.jpl.nasa.gov/), are used. The emissivities in the two split-window channels of MODIS ( $\varepsilon_{31}$ and $\varepsilon_{32}$ ) and S-VISSR ( $\varepsilon_{I R 1}$ and $\left.\varepsilon_{I R 2}\right)$ were calculated by the integrals of the spectral emissivity with the channel response functions over the spectral range of the channels. The channel response functions of the two split-window channels for MODIS and FY-2C are displayed respectively in figure 5 .

A statistical relationship between MODIS channels and S-VISSR channels was established by a linear regression analysis. As a result, the emissivities in S-VISSR channels IR1 and IR2 are, respectively, related to the emissivities in MODIS channels 31 and 32 by Eqs. (4) and (5).

$$
\begin{aligned}
& \varepsilon_{I R 1}=-0.0611+1.0614 \varepsilon_{31} \\
& \varepsilon_{I R 2}=-0.0210+1.0199 \varepsilon_{32}
\end{aligned}
$$




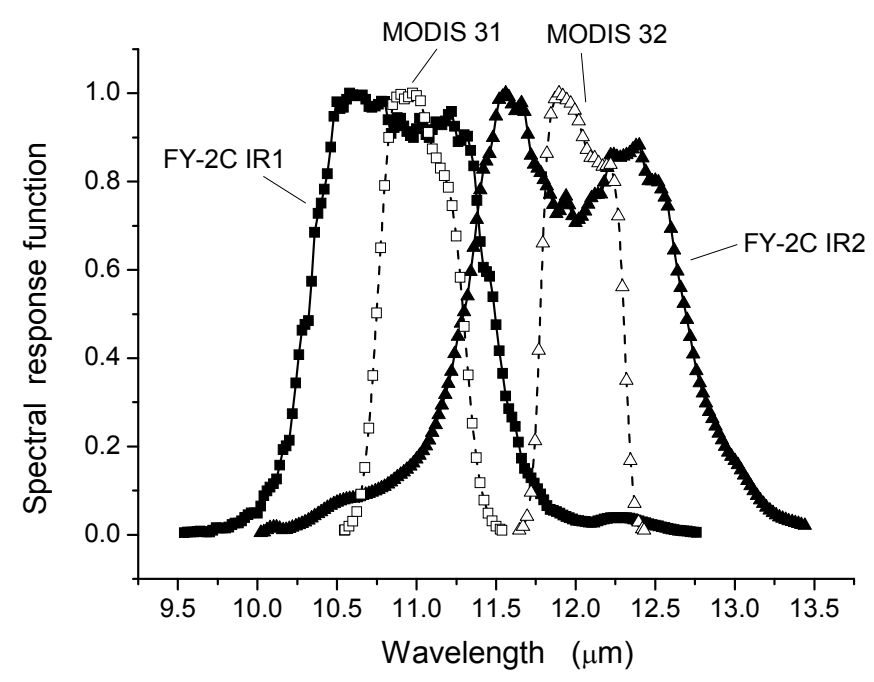

Figure 5. S-VISSR and MODIS split-window spectral response functions.

Figure 6 shows the emissivities and linear regression results. Only the emissivities of soil, vegetation, water, and snow/ice in JHU and UCSB databases were included in this work. Some few deviated points in this figure are due to the fact that the spectral ranges of S-VISSR channels IR1 and IR2 are broader than those of MODIS channels 31 and 32 as shown in figure 5. However, as shown in figure 6 , the results of the linear regression are good with the RMSEs within 0.002, which indicates that the emissivities in S-VISSR channels IR1 and IR2 can be directly derived from those in MODIS channels 31 and 32, respectively.
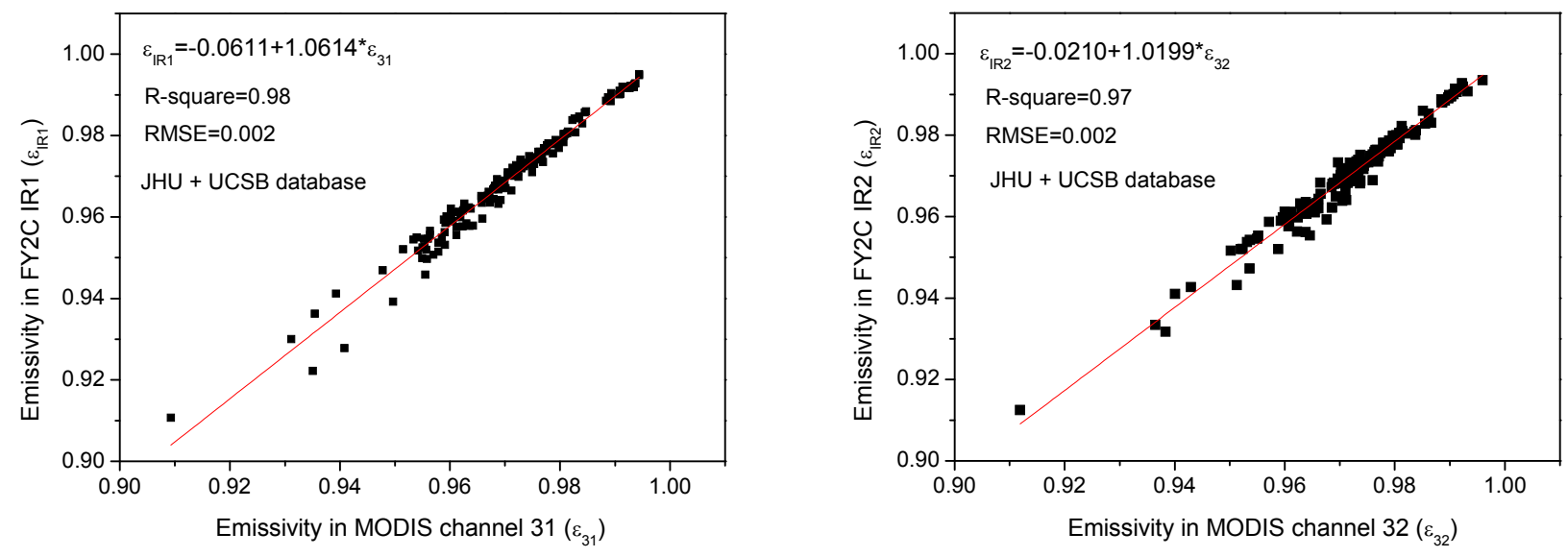

Figure 6. Linear fitting relationship of the emissivities between the S-VISSR channels IR1 and IR2 and the MODIS channels 31 and 32, respectively.

Alternatively, the emissivities of the S-VISSR IR1 and IR2 channels can be estimated either with the land surface classification as did by Sun and Pinker (2003) [13] or using the method developed by Jiang et al. (2006) [26] which combined mid-infrared and thermal infrared data of SEVIRI to retrieve LSE. 


\subsection{Determination of the atmospheric WVC}

The MODIS total precipitable water product MOD05 provides the atmospheric column water vapor amounts, which can be used as the model input when the scanning times of the sensors MODIS and S-VISSR are close to each other. However, MODIS provides the instantaneous WVC only four times per day, which can not meet the need for the temporal resolutions (an hour) of S-VISSR onboard FY2C. Since the atmospheric WVC changes with time, the method developed by Li et al. (2003) [27] can be used to determine the WVC from S-VISSR IR1 and IR2 data.

According to Li et al. (2003) [27], the atmospheric WVC can be derived by the use of the transmittance ratio of split-window channels,

with

$$
W V C=c_{1}+c_{2} \times \frac{\tau_{j}}{\tau_{i}}
$$

and

$$
\begin{gathered}
\frac{\tau_{j}}{\tau_{i}}=\frac{\varepsilon_{i}}{\varepsilon_{j}} R_{j i} \\
R_{j i}=\frac{\sum_{k=1}^{N}\left(T_{i, k}-\overline{T_{i}}\right)\left(T_{j, k}-\overline{T_{j}}\right)}{\sum_{k=1}^{N}\left(T_{i, k}-\bar{T}_{i}\right)^{2}}
\end{gathered}
$$

where $c_{1}$ and $c_{2}$ are unknown coefficients, $\tau_{i}$ and $\tau_{j}$ are the atmospheric transmittances in the splitwindow channels $i$ and $j$, the subscript $k$ denotes pixel $k$, and the $\bar{T}_{i}$ and $\bar{T}_{j}$ are the TOA mean (or the median) channel brightness temperatures of the $N$ neighboring pixels considered for channels $i$ and $j$, respectively.

On the basis of the numerical results obtained in Section 2.2, coefficients $c_{1}$ and $c_{2}$ can be respectively derived as functions of secant VZA as

$$
\begin{aligned}
& c_{1}=28.104-14.996 / \cos (V Z A)+3.211 / \cos ^{2}(V Z A) \\
& c_{2}=-28.056+14.954 / \cos (V Z A)-3.206 / \cos ^{2}(V Z A)
\end{aligned}
$$

Figure 7 shows the curve fits of the coefficients $c_{1}, c_{2}$ as functions of secant VZA. As noted, the fitting results are quit well with both R-squares equal to 0.999. In addition, with the actual WVC and the transmittance ratio of split-window channels IR1 and IR2 obtained in Section 2.2, the RMSE between the actual WVC and the WVC estimated using Eqs. (6), (9) and (10) is $0.17 \mathrm{~g} / \mathrm{cm}^{2}$, which indicates that the fitting results are good.

\subsection{Sensitivity analysis}

As Wan and Dozier (1996) [21] indicated that the errors of LST estimated by the GSW algorithm come mainly from the uncertainties of LSEs, atmospheric properties and the instrument noises. These three uncertainties of error are taken into account in this investigation. 

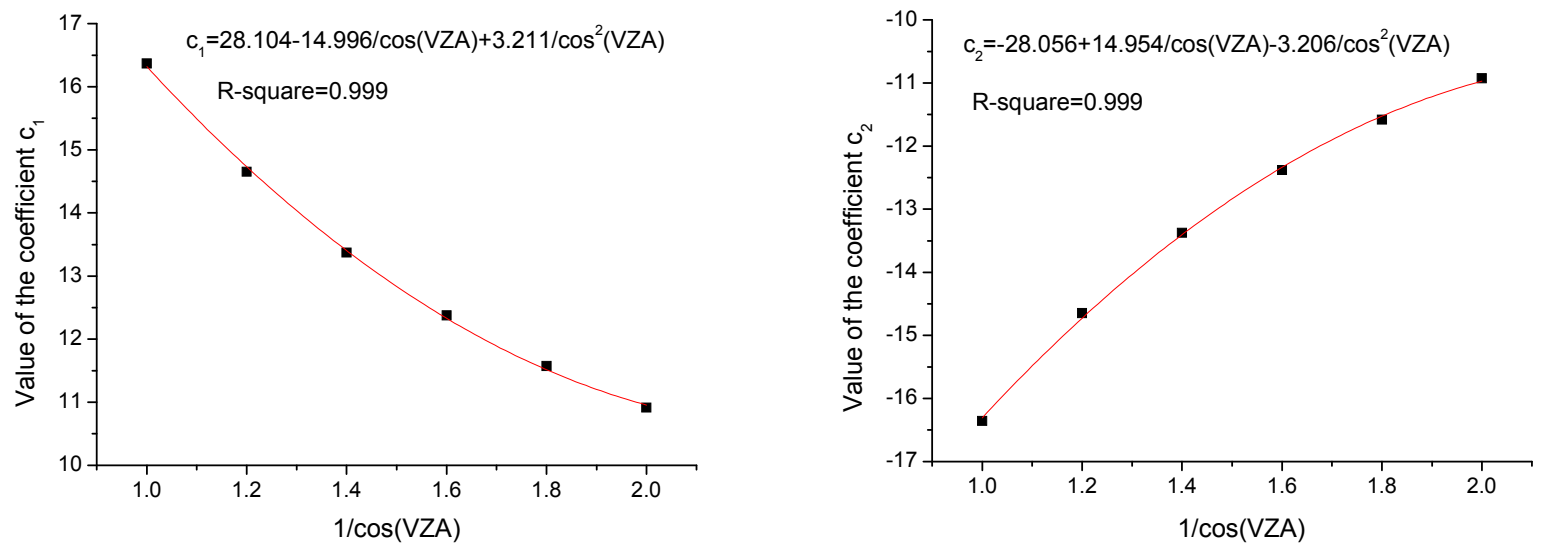

Figure 7. Curve fits of the coefficients $c_{1}-c_{2}$ in Eq. (6) as functions of the VZA

\subsubsection{Sensitivity analysis to instrumental noises (NE $\Delta \mathrm{T})$}

In order to see how significant the effect of the instrumental NE $\Delta \mathrm{T}$ on the retrieval of LST, a Gaussian random distribution error of $0.1 \mathrm{~K}, 0.2 \mathrm{~K}$ and $0.5 \mathrm{~K}$ are, respectively added to the TOA brightness temperatures $T_{i}$ and $T_{j}$ in Eq. (3). Then we estimate the LST using GSW algorithm with the noised TOA brightness temperatures. As an example, compared the actual LST with the estimated LST for the sub-range: $\varepsilon \in[0.94,1.0], W V C \in[1.0,2.5]$, and $T_{s} \in[290 K, 310 K]$, the RMSE is $0.38 \mathrm{~K}$ for $\mathrm{NE} \Delta \mathrm{T}=0.1 \mathrm{~K}, 0.43 \mathrm{~K}$ for $\mathrm{NE} \Delta \mathrm{T}=0.2 \mathrm{~K}$, and $0.67 \mathrm{~K}$ for $\mathrm{NE} \Delta \mathrm{T}=0.5 \mathrm{~K}$. Compared the RMSE of 0.37 $\mathrm{K}$ for no instrumental noise, the accuracy of retrieval LST can be affected by $3 \%$ for NE $\Delta \mathrm{T}=0.1 \mathrm{~K}$, by $16 \%$ for $\mathrm{NE} \Delta \mathrm{T}=0.2 \mathrm{~K}$, and by $81 \%$ for $\mathrm{NE} \Delta \mathrm{T}=0.5 \mathrm{~K}$.

\subsubsection{Sensitivity analysis to LSEs}

According to the Eq. (3), the sensitivity of the uncertainties in LSEs is mainly dependent on the terms $(1-\varepsilon) / \varepsilon$ and $\Delta \varepsilon /\left(\varepsilon^{2}\right)$, which can be written as

$$
\begin{aligned}
& \alpha=a_{2} \frac{T_{i}+T_{j}}{2}+a_{5} \frac{T_{i}-T_{j}}{2} \\
& \beta=a_{3} \frac{T_{i}+T_{j}}{2}+a_{6} \frac{T_{i}-T_{j}}{2}
\end{aligned}
$$

Two cases are considered in this investigation. One is the extremely dry atmospheric condition $(W V C \in[0.0,1.5])$ and the other is the extremely wet atmospheric condition $(W V C \in[5.0,6.5])$. With the regression coefficients and the $T_{i}$ and $T_{j}$ simulated in Section 2.2, using equations (11) and (12) we can obtain the variations of $\alpha$ and $\beta$. Table 2 lists the variations of $\alpha$ and $\beta$ for the sub-range: $\varepsilon \in[0.94,1.0], T_{s} \in[290 K, 310 K], W V C \in[0.0,1.5]$ and the sub-range $\varepsilon \in[0.94,1.0]$, $T_{s} \in[290 K, 310 K], W V C \in[5.0,6.5]$, for $\mathrm{VZA}=0^{\circ}$, respectively. 
Table 2. Statistics of the errors due to the uncertainties in LSEs for the subrange $\varepsilon \in[0.94,1.0], T_{s} \in[290 K, 310 K], W V C \in[0.0,1.5]$ and the sub-range $\varepsilon \in[0.94,1.0]$, $T_{s} \in[290 K, 310 K], W V C \in[5.0,6.5]$, for $\mathrm{VZA}=0^{\circ}$.

\begin{tabular}{|l|l|l|l|l|}
\hline Conditions & \multicolumn{4}{l}{$\varepsilon \in[0.94,1.0], T_{s} \in[290 K, 310 K], \mathrm{VZA}=0^{\circ}$} \\
\hline $\begin{array}{l}\text { Water vapor content } \\
\left(\mathbf{g} / \mathbf{c m}^{2}\right)\end{array}$ & \multicolumn{2}{|l|}{$W V C \in[0.0,1.5]$} & \multicolumn{2}{l|}{$W V C \in[5.0,6.5]$} \\
\hline Variable & $\alpha$ & $\beta$ & $\alpha$ & $\beta$ \\
\hline Range of Values (K) & {$[44.80,61.23]$} & {$[-135.71,-121.05]$} & {$[11.57,34.42]$} & {$[-70.13,-19.48]$} \\
\hline Mean (K) & 52.39 & -127.60 & 23.29 & -45.56 \\
\hline $\begin{array}{l}\text { Standard deviation } \\
(\text { K) }\end{array}$ & 3.10 & 3.06 & 4.22 & 9.32 \\
\hline
\end{tabular}

From table 2 one can see that the values of $\alpha$ and $\beta$ in extremely dry atmospheric condition $(W V C \in[0.0,1.5])$ are nearly two times as large as those of $\alpha$ and $\beta$ in extremely wet atmospheric condition, $(W V C \in[5.0,6.5])$, respectively. This means that the sensitivity of $(1-\varepsilon) / \varepsilon$ and $\Delta \varepsilon /\left(\varepsilon^{2}\right)$ to LST for wet atmospheric condition is decreased two times as that for dry atmospheric condition.

From equation (3), the LST error $\delta L S T$ due to the uncertainty in $(1-\varepsilon) / \varepsilon$ and $\Delta \varepsilon /\left(\varepsilon^{2}\right)$ can be estimated by,

$$
\delta L S T=\sqrt{\alpha^{2} \delta\left(\frac{1-\varepsilon}{\varepsilon}\right)^{2}+\beta^{2} \delta\left(\frac{\Delta \varepsilon}{\varepsilon^{2}}\right)^{2}}
$$

Assuming that the uncertainties of $(1-\varepsilon) / \varepsilon$ and $\Delta \varepsilon /\left(\varepsilon^{2}\right)$ are around $1 \%$, the LST error is [1.3K, $1.5 \mathrm{~K}]$ with the mean of $1.4 \mathrm{~K}$ for the dry atmosphere and $[0.2 \mathrm{~K}, 0.8 \mathrm{~K}]$ with the mean of $0.5 \mathrm{~K}$ for the wet atmosphere.

\subsubsection{Sensitivity analysis to the atmospheric WVC}

It is well known that the WVC in the atmosphere is not easily determined from satellite data. In order to see how significant the effect of the uncertainty of the WVC on the retrieval of LST in GSW algorithm, the wrong sub-range selection of the WVC is investigated in our work. As mentioned above in Section 3.1, the WVC was divided into six sub-ranges with an overlap of $0.5 \mathrm{~g} / \mathrm{cm}^{2}$. The overlap WVC could be fallen into two adjacent sub-ranges. That is, it is included by two sub-ranges and corresponded to two pairs of coefficients $a_{0}-a_{6}$. We aim to analyze the effect of the overlap WVC on the retrieval of LST.

Figure 8 gives an example of the uncertainty of the WVC. From figure 8 one can see that the overlap water vapor content $W V C \in[1.0,1.5]$ falling into two sub-ranges $W V C \in[0.0,1.5]$ and $W V C \in[1.0,2.5]$. When we estimate the LST with the water vapor content $W V C \in[1.0,1.5]$ using the coefficients corresponding to the sub-range $\varepsilon \in[0.94,1.0], W V C \in[0.0,1.5]$, and $T_{s} \in[290 \mathrm{~K}, 310 \mathrm{~K}]$, the RMSE between the actual and the estimated $T_{s}$ is $0.18 \mathrm{~K}$, while using the coefficients corresponding to the sub-range $\varepsilon \in[0.94,1.0], W V C \in[1.0,2.5]$, and $T_{s} \in[290 K, 310 K]$, the RMSE is $0.43 \mathrm{~K}$. 

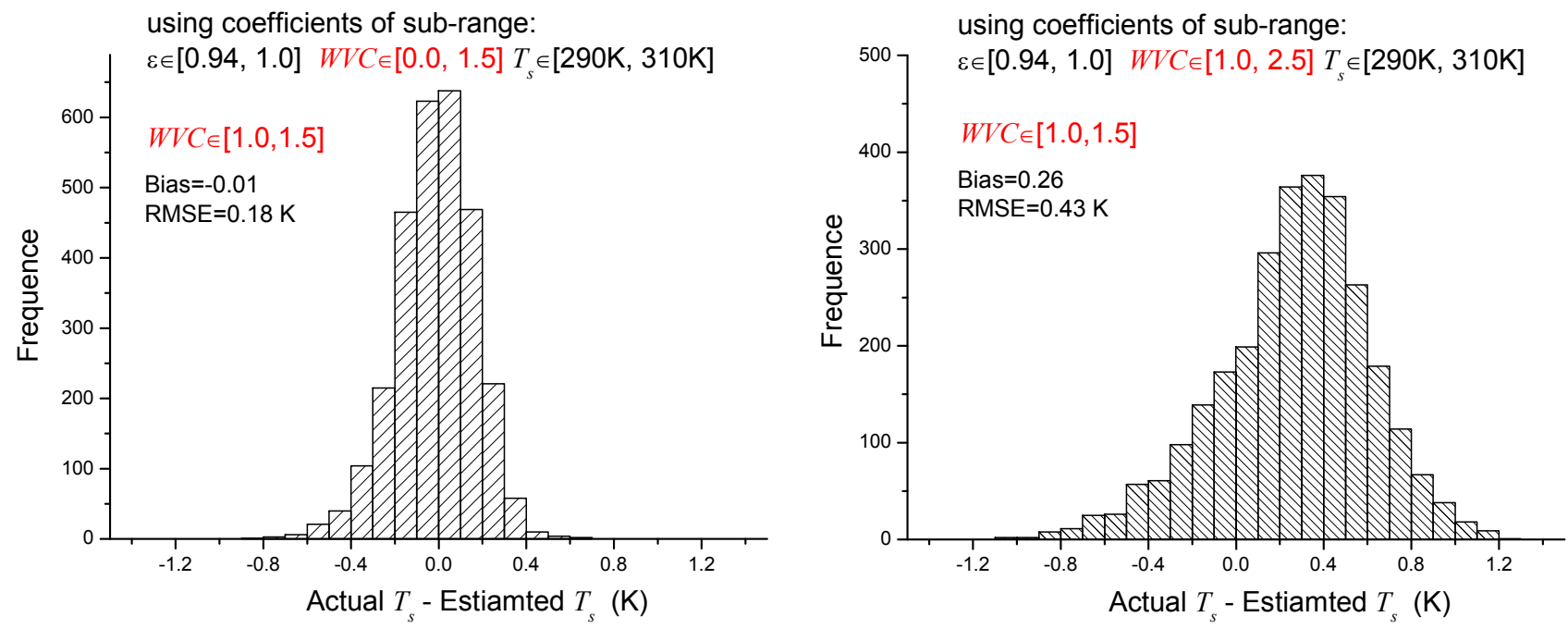

Figure 8. Histogram of the difference between the actual and estimated $T_{s}$ for the overlap water vapor content $W V C \in[1.0,1.5]$ using the coefficients of different sub-ranges.

\subsection{Intercomparison of different formulations of the split-window algorithms}

It is well known that the LST retrieval from satellite observations has been ongoing for several decades. Many different formulations of the split-window algorithms have been proposed. They are somewhat similar in formulation and several of them are directly inspired from Becker and Li's (1990) [12] formulation. In order to perform the intercomparison with the recently proposed split-window algorithms, different formulations were used to estimate the LST with the same simulated FY-2C data in this work. Those formulations are listed in table 3:

Table 3. Different formulations of split-window algorithms in literatures, $\varepsilon$ is the averaged emissivity in channesl $i$ and $j$, and $\Delta \varepsilon$ is the difference between the two channels emissivities.

\begin{tabular}{|l|l|}
\hline Authors & Formulations \\
\hline Price, 1984 [8] & $T_{s}=a_{0}+a_{1} T_{i}+a_{2}\left(T_{i}-T_{j}\right)+a_{3}\left(T_{i}-T_{j}\right)(1-\varepsilon)+a_{4} T_{j} \Delta \varepsilon$ \\
\hline Prata and Platt, 1991 [14] & $T_{s}=a_{0}+a_{1} \frac{T_{i}}{\varepsilon}+a_{2} \frac{T_{j}}{\varepsilon}+a_{3} \frac{1-\varepsilon}{\varepsilon}$ \\
\hline Vidal, 1991 [15] & $T_{s}=a_{0}+a_{1} T_{i}+a_{2}\left(T_{i}-T_{j}\right)+a_{3} \frac{1-\varepsilon}{\varepsilon}+a_{4} \frac{\Delta \varepsilon}{\varepsilon}$ \\
\hline Ulivieri et al., 1992 [16] & $T_{s}=a_{0}+a_{1} T_{i}+a_{2}\left(T_{i}-T_{j}\right)+a_{3}(1-\varepsilon)+a_{4} \Delta \varepsilon$ \\
\hline Sobrino et al., 1993 [17] & $T_{s}=a_{0}+a_{1} T_{i}+a_{2}\left(T_{i}-T_{j}\right)+a_{3}\left(T_{i}-T_{j}\right)^{2}+a_{4}(1-\varepsilon)+a_{5} \Delta \varepsilon$ \\
\hline Sobrino et al., 1994 [18] & $T_{s}=a_{0}+a_{1} T_{i}+a_{2}\left(T_{i}-T_{j}\right)+a_{3} \varepsilon+a_{4} \frac{\Delta \varepsilon}{\varepsilon}$ \\
\hline Coll et al., 1997 [19] & $T_{s}=T_{i}+a_{0}+a_{1}\left(T_{i}-T_{j}\right)+a_{2}\left(T_{i}-T_{j}\right)^{2}+a_{3}(1-\varepsilon)+a_{4} \Delta \varepsilon$ \\
\hline
\end{tabular}


In addition, Becker and Li (1995) [20] further modified their split-window algorithm (Becker and $\mathrm{Li}, 1990)$ [12] by adding atmospheric water vapor correction as

$$
T_{s}=A_{0}+P \frac{T_{i}+T_{j}}{2}+M \frac{T_{i}-T_{j}}{2}
$$

with

$$
\begin{aligned}
& A_{0}=a_{0}+a_{1} w \\
& P=a_{2}+\left(a_{3}+a_{4} w \cos \theta\right)(1-\varepsilon)-\left(a_{5}+a_{6} w\right) \Delta \varepsilon \\
& M=a_{7}+a_{8} w+\left(a_{9}+a_{10} w\right)(1-\varepsilon)-\left(a_{11}+a_{12} w\right) \Delta \varepsilon
\end{aligned}
$$

where $\varepsilon=\left(\varepsilon_{i}+\varepsilon_{j}\right) / 2$ and $\Delta \varepsilon=\varepsilon_{i}-\varepsilon_{j}, w$ is the total precipitable water amount, and $\theta$ is the Viewing Zenith Angle (VZA).

In order to make the intercomparsion more reasonable, the coefficients from the above equations have been recalculated using the same simulated FY-2C data within the same sub-ranges in Section 3.1. As an example, table 4 depicts the RMSEs between the actual and the estimated $T_{s}$ versus the secant VZA for the sub-range: $\varepsilon \in[0.94,1.0], W V C \in[1.0,2.5]$, and $T_{s} \in[290 K, 310 K]$. From this table, one can see that the RMSEs increase with the increase of the VZA for all algorithms. In addition, except for the algorithms proposed by Price (1984) [8], and Prata and Platt (1991) [14], the $T_{s}$ estimated using the other algorithms are comparable, which indicates that the split-window algorithm can be successfully applied to the LST retrievals from FY-2C data. It should be noted that the RMSE values in Table 4 will be larger, especially for BL95, in considering the sensitivity to WVC errors.

\begin{tabular}{|c|c|c|c|c|c|c|c|c|c|c|}
\hline \multirow{8}{*}{$\begin{array}{c}\text { RMSE } \\
\text { (K) }\end{array}$} & & \multicolumn{9}{|c|}{ Authors } \\
\hline & $\operatorname{VZA}\left({ }^{\circ}\right)$ & GSW & Price84 & Prata91 & Vidal91 & Ulivieri92 & Sobrino93 & Sobrino94 & Coll97 & BL95 \\
\hline & 0 & 0.37 & 0.73 & 1.15 & 0.38 & 0.38 & 0.37 & 0.38 & 0.38 & 0.22 \\
\hline & 33.56 & 0.41 & 0.74 & 1.26 & 0.43 & 0.42 & 0.42 & 0.42 & 0.43 & 0.25 \\
\hline & 44.42 & 0.46 & 0.74 & 1.35 & 0.48 & 0.47 & 0.47 & 0.47 & 0.47 & 0.28 \\
\hline & 51.32 & 0.52 & 0.75 & 1.43 & 0.53 & 0.53 & 0.51 & 0.53 & 0.52 & 0.32 \\
\hline & 56.25 & 0.57 & 0.77 & 1.49 & 0.58 & 0.58 & 0.57 & 0.58 & 0.57 & 0.36 \\
\hline & 60 & 0.63 & 0.80 & 1.54 & 0.64 & 0.64 & 0.62 & 0.64 & 0.62 & 0.41 \\
\hline
\end{tabular}

Table 4. RMSEs between the actual $T_{s}$ and the $T_{s}$ estimated using different formulations of the split-window algorithms for the sub-range $\varepsilon \in[0.94,1.0], W V C \in[1.0,2.5]$, and $T_{s} \in[290 K, 310 K]$.

\section{Application to actual FY-2C satellite data}

The objective of the present work is to estimate the LST from Chinese first operational geostationary meteorological satellite FengYun-2C (FY-2C) data for cloud-free skies. Figure 9 gives an example of the retrieval LST around Beijing in China during FY-2C satellite scanning on May 15, 2006 at 11:00 local time. The model inputs are the TOA brightness temperatures LSTs, VZA, LSEs, 
and WVC. The TOA brightness temperatures LSTs and VZA are directly extracted from the FY-2C satellite data. The LSEs are derived from the emissivities in MODIS channels 31 and 32 provided by MODIS/Terra LST product MOD11B1, and the WVC are obtained from MODIS total precipitable water product MOD05. Symbols A, B, and C located in red, green and baby blue colored areas in figure 9 represent bare soil, cultivated surface and sea surface, respectively.

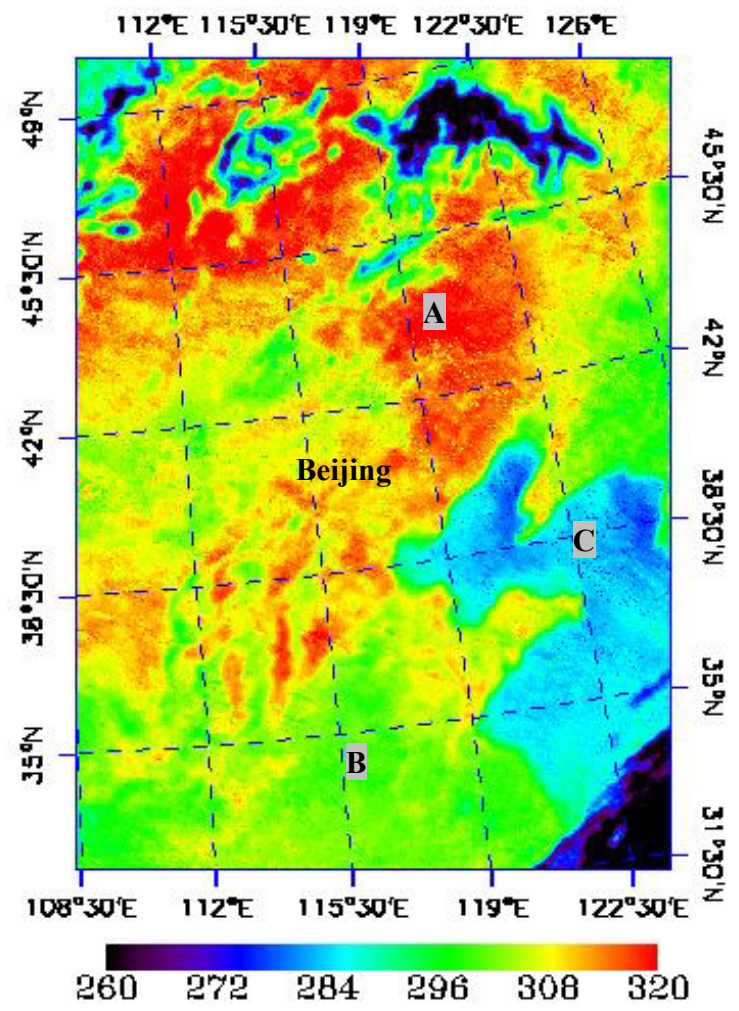

Figure 9. Map of the LST estimated from FY-2C satellite data at 11:00 local time on May $15,2006$.

In addition, table 5 lists the values of the VZA, WVC, LSE, TOA brightness temperature, and resultant $T_{s}$ for one representative pixel in each red, green, and baby blue colored areas in figure 9 .

Table 5. Description of symbols A, B and C in figure 9.

\begin{tabular}{|l|l|l|l|}
\hline & A (red) & B (green) & C (baby blue) \\
\hline Longitude $\left(^{\circ}\right)$ & $120.06 \mathrm{E}$ & $116.15 \mathrm{E}$ & $122.75 \mathrm{E}$ \\
\hline Latitude $\left(^{\circ}\right)$ & $43.70 \mathrm{~N}$ & $33.84 \mathrm{~N}$ & $38.47 \mathrm{~N}$ \\
\hline $\mathrm{VZA}\left(^{\circ}\right)$ & 53.44 & 41.96 & 49.14 \\
\hline $\mathrm{WVC}\left(\mathrm{g} / \mathrm{cm}^{2}\right)$ & 0.868 & 1.465 & 1.217 \\
\hline$\varepsilon_{I R 1}$ & 0.944 & 0.962 & 0.986 \\
\hline$\varepsilon_{I R 2}$ & 0.946 & 0.966 & 0.99 \\
\hline$T_{I R 1}(\mathrm{~K})$ & 309.42 & 295.24 & 281.95 \\
\hline$T_{I R 2}(\mathrm{~K})$ & 307.32 & 294.58 & 282.20 \\
\hline$T_{s}(\mathrm{~K})$ & 318.35 & 299.74 & 286.47 \\
\hline
\end{tabular}


It should be pointed out here that the LST estimated from the FY-2C satellite data has not been validated with in situ measurements since there are no in situ measurements available. In addition, due to the extreme difficulty or impossibility to get the LST at ground level representative at $5 \mathrm{~km} * 5 \mathrm{~km}$, we will try to cross validate LST derived from FY-2C data in the future with the well validated LST product provided by MODIS data.

\section{Conclusions}

In this paper, we have addressed the retrieval of the Land Surface Temperature (LST) from the Chinese first operational geostationary meteorological satellite FengYun-2C (FY-2C) data in two thermal infrared channels IR1 (10.3-11.3 $\mu \mathrm{m})$ and IR2 (11.5-12.5 $\mu \mathrm{m})$, using the Generalized Split-Window (GSW) algorithm proposed by Wan and Dozier (1996) [21].

Taking into account the fact that the S-VISSR sensor onboard FY-2C has no atmospheric sounding channels, the coefficients in the GSW algorithm were derived by dividing the ranges of the mean emissivity, the atmospheric Water Vapor Content (WVC), and the LST into tractable sub-ranges, and were recalculated using a statistical regression method from the numerical values simulated with an accurate atmospheric radiative transfer model MODTRAN 4 over a wide range of the atmospheric and surface conditions. The simulation analysis showed that the LST could be estimated by the GSW algorithm with the Root Mean Square Error (RMSE) less than $1 \mathrm{~K}$ for the sub-ranges with the Viewing Zenith Angle (VZA) less than $30^{\circ}$ or for the sub-ranges with VZA less than $60^{\circ}$ and the atmospheric WVC less than $3.5 \mathrm{~g} / \mathrm{cm}^{2}$ provided that the Land Surface Emissivities (LSEs) are known.

As the GSW algorithm requires WVC and LSE as model input, the MODIS total precipitable water product MOD05 providing the atmospheric column water vapor amounts, was used to obtain the WVC when the scanning times of the sensors MODIS and S-VISSR are close to each other. As for the other scanning times of S-VISSR, the atmospheric WVC can be determined using the method developed by Li et al. (2003) [27]. As for LSE, the MODIS/Terra LST product MOD11B1 providing the LSEs with $5 \mathrm{~km}$ resolution for the thermal infrared channels 31 and 32, was used to derive the LSEs in S-VISSR channels IR1 and IR2, respectively.

In addition, the sensitivity and error analyses in term of the uncertainty of the LSE and WVC as well as the instrumental noise were also performed in this work. The results show that the accuracy of retrieval LST can be affected by $3 \%$ for $\mathrm{NE} \Delta \mathrm{T}=0.1 \mathrm{~K}$, by $16 \%$ for $\mathrm{NE} \Delta \mathrm{T}=0.2 \mathrm{~K}$, and by $81 \%$ for $\mathrm{NE} \Delta \mathrm{T}=0.5 \mathrm{~K}$ for the sub-range $\varepsilon \in[0.94,1.0], W V C \in[1.0,2.5]$, and $T_{s} \in[290 \mathrm{~K}, 310 \mathrm{~K}]$; given the uncertainties of $(1-\varepsilon) / \varepsilon$ and $\Delta \varepsilon /\left(\varepsilon^{2}\right)$ around $1 \%$, the LST error is $[1.3 \mathrm{~K}, 1.5 \mathrm{~K}]$ with the mean of $1.4 \mathrm{~K}$ for the dry atmosphere and $[0.2 \mathrm{~K}, 0.8 \mathrm{~K}]$ with the mean of $0.5 \mathrm{~K}$ for the wet atmosphere; and the effect of the uncertainty of the WVC on the retrieval LST could be around $0.3 \mathrm{~K}$.

Moreover, in order to compare the different formulations of the split-window algorithm, several split-window algorithms were used to estimate the LST with the same simulated FY-2C data. The result of the intercomparsion showed that most of the algorithms give comparable results, which indicates that the split-window algorithm can be successfully applied to the LST retrievals from FY-2C data. 


\section{Acknowledgements}

This work was supported by the National Natural Science Foundation of China under Grant 40425012 and the "Hundred Talent" program of the Chinese Academy of Science.

\section{References and Notes}

1. Mannstein, H; Surface energy budget, surface temperature, and thermal inertia. In Remote Sensing Applications in Meteorology and Climatology; Vaughan, R.A., Reidel, D., Eds. Dordrecht: Netherlands, 1987 (NATO ASI Ser. C: Math. Phys. Sci., vol. 201).

2. Sellers, P.J.; Hall, F.G.; Asrar, G.; Strebel, D.E.; Murphy, R.E. The first ISLSCP Field Experiment (FIFE). Bullet of American Meteorology Society 1988, 69(1), 22-27.

3. Serafini, V.V. Estimation of the evapotranspiration using surface and satellite data. International journal of remote sensing 1987, 8, 1547-1562.

4. Bussieres, No.; Louie, P.Y.T.; Hogg, W. Progress report on the implementation of an algorithm to estimate regional evaportanspiration using satellite data. In Proceeding of the workshop on applications of remote sensing in hydrology, Saskaton Saskatchewan, 13-14 February, 1990.

5. Price, J.C. The potential of Remotely Sensed Thermal Infrared data to Infer Surface Soil Moisture and Evaporation. Water Resources 1990, 16, 787-795.

6. Schmugge, T.J.; André, J.C. Land Surface Evaporation: Measurements and Parameterization. Springer-Verlag: New York, 1991.

7. Running, S.W.; Justice, C.; Salomonson, V.; Hall, D.; Barker, J.; Kaufman, Y.; Strahler, A.; Huete, A.; Muller, J.-P.; Vanderbilt, V.; Wan, Z.; Teillet, P. Terrestrial remote sensing science and algorithms planned for EOS/MODIS. International journal of remote sensing 1994, 17, 35873620 .

8. Price, J.C. Land surface temperature measurements from the split window channels of the NOAA 7 AVHRR. Journal of Geophysical research 1984, D5, 7231-7237.

9. Ottlé, C.; Vidal-Madjar, D. Estimation of land surface temperature with NOAA9 data. Remote Sensing of Environment 1992, 40(1), 27-41.

10. Jiménez-Muñoz, J.C.; Sobrino, J.A. A generalized single-channel method for retrieving land surface temperature from remote sensing data. Journal of Geophysical Research 2003, 108(D22), 4688; DOI: 10.1029/2003JD003480.

11. McMillin, L.M. Estimation of sea surface temperature from two infrared window measurements with different absorption. Journal of Geophysical Research 1975, 80, 5113-5117.

12. Becker, F.; Li, Z.-L. Toward a local split window method over land surface. International Journal of Remote Sensing 1990, 3, 369-393.

13. Sun, D.; Pinker, R.T. Estimation of land surface temperature from Geostationary Operational Environmental Satellite (GOES-8). Journal of Geophysical Research 2003, 108(D11), 4326; DOI: 10.1029/2002JD002422.

14. Prata, A.J.; Platt, C.M.R. Land surface temperature measurements from the AVHRR. In Proceedings of the $5^{\text {th }}$ AVHRR data users conference, Tromso, Norway, June 25-28, 1991; EUM P09, pp. 443-438. 
15. Vidal, A. Atmospheric and emissivity correction of land surface temperature measured from satellite using ground measurements or satellite data. International Journal of Remote Sensing 1991, 12, 2449-2460.

16. Ulivieri, C.; Castronouvo, M.M.; Francioni, R.; Cardillo, A. A SW algorithn for estimating land surface temperature from satellites. Advance Space Research 1992, 14(3), 59-65.

17. Sobrino, J.A.; Li, Z.-L.; Stoll, M.P.; Becker, F. Determination of the surface temperature from ATSR data. In Proceedings of $25^{\text {th }}$ International Symposium on Remote Sensing of Environment, Graz, Austria, April 4-8, 1993; pp. II-19 - II-109.

18. Sobrino, J.A.; Li, Z.-L.; Stoll, M.P.; Becker, F. Improvements in the split-window technique for land surface temperature determination. IEEE Transections on Geoscience and Remote Sensing 1994, 32(2), 243-253.

19. Coll C.; Caselles, V. A split-window algorithm for land surface temperature from advanced very high resolution radiometer data: Validation and algorithm comparison. Journal of Geophysical research Atmospheres 1997, 102(D14), 16697-16713.

20. Becker, F.; Li, Z.-L. Surface temperature and emissivity at various scales: Definition, measurement and related problems. Remote Sensing of Environment 1995, 12, 225-253.

21. Wan, Z.; Dozier, J. A generalized split-window algorithm for retrieving land-surface temperature from space. IEEE Transections on Geoscience and Remote Sensing 1996, Vol.34, No.4, 892-905.

22. Sobrino, J.A; Romaguera, M. Land surface temperature retrieval from MSG1-SEVIRI data. Remote Sensing of Environment 2004, 92, 247-254.

23. Li, Z.-L.; Petitcolin, F.; Zhang, R.H. A physically based algorithm for land surface emissivity retrieval from combined mid-infrared and thermal infrared data. Science in China (Series E) 2000, 43, 23-33.

24. Berk, A.; Bernstein, L.S.; Anderson, G.P.; Acharya, P.K.; Robertson, D.C.; Chetwynd, J.H.; et al. MODTRAN cloud and multiple scattering upgrades with application to AVHRIS. Remote Sensing of Environment 1998, 65, 367-375.

25. Scott, N.A.; Chédin, A. A fast line-by-line method for atmospheric absorption computations: The Automatized Atmospheric Absorption Atlas. Journal of Applied Meteorology 1981, 20, 802-812.

26. Jiang, G.-M.; Li, Z.-L.; Nerry, F. Land surface emissivity retrieval from combined mid-infrared and thermal infrared data of MSG-SEVIRI. Remote Sensing of Environment 2006, 105, 326-340.

27. Li, Z.-L.; Jia, L.; Su, Z.; Wan, Z.; Zhang, R. A new approach for retrieving precipitable water from ATSR2 split-window channel data over land area. International Journal of Remote Sensing 2003, 24(24), 5095-5117.

(C) 2008 by MDPI (http://www.mdpi.org). Reproduction is permitted for noncommercial purposes. 